\title{
High-quality spectrophotometry of the planetary nebula in the Fornax dSph ${ }^{\star}$
}

\author{
A. Y. Kniazev ${ }^{1,2,3}$, E. K. Grebel ${ }^{4,5}$, S. A. Pustilnik ${ }^{3}$, and A. G. Pramskij ${ }^{3}$ \\ 1 European Southern Observatory, Karl-Schwarzschild-Strasse 2, 85748 Garching, Germany \\ e-mail: akniazev@saao.ac.za \\ 2 Current address: South African Astronomical Observatory, Observatory Road, Cape Town, South Africa \\ Special Astrophysical Observatory, Nizhnij Arkhyz, Karachai-Circassia 369167 Russia \\ 4 Astronomical Institute of the University of Basel, Department of Physics and Astronomy, Venusstrasse 7, 4102 Binningen, \\ Switzerland \\ 5 Astronomisches Rechen-Institut, Zentrum für Astronomie Heidelberg, University of Heidelberg, Mönchhofstr. 12-14, \\ 69120 Heidelberg, Germany
}

Received 26 January 2006 / Accepted 16 March 2007

\section{ABSTRACT}

\begin{abstract}
We present results of NTT spectroscopy of the one known planetary nebula (PN) in the dwarf spheroidal galaxy Fornax, a gasdeficient Local Group galaxy that stopped its star formation activity a few hundred million years ago. We detected the [O III] $\lambda 4363$ line with a signal-to-noise ratio of $\sim 22$. For the first time we detected the weak [S II] $\lambda \lambda 6717,6731$ lines $(I(6717+6731) \approx 0.01 I(\mathrm{H} \beta))$, determined the electron number density $\left(N_{\mathrm{e}}(\mathrm{SII})=750 \mathrm{~cm}^{-3}\right)$, and calculated $\mathrm{O}, \mathrm{N}, \mathrm{Ne}, \mathrm{Ar}, \mathrm{S}, \mathrm{Cl}, \mathrm{Fe}, \mathrm{He}$ and $\mathrm{C}$ abundances. The abundance analysis presented here is based on the direct calculation of the electron temperature $T_{\mathrm{e}}$ and yields an oxygen abundance of $12+\log (\mathrm{O} / \mathrm{H})=8.28 \pm 0.02$. The analysis of the $\mathrm{O}, \mathrm{Ne}, \mathrm{Ar}$ and $\mathrm{S}$ abundances shows that the original ISM oxygen abundance was $0.27 \pm 0.10$ dex lower and that third-dredge-up self-pollution in oxygen took place. The blue spectrum shows weak Wolf-Rayet features, and the progenitor star is classified as a weak emission-line star. Four of the five PNe in dwarf spheroidal galaxies are now known to show WR wind features. Overall, the metallicity of the progenitor of the PN fits in well with stellar spectroscopic abundances derived in previous studies as well as with the stellar age-metallicity relation of Fornax.
\end{abstract}

Key words. galaxies: abundances - galaxies: evolution - galaxies: individual: Fornax - planetary nebulae: individual: Fornax

\section{Introduction}

Understanding how the elemental abundances of galaxies have changed over time is an essential issue for understanding galaxy evolution. Abundance measurements constrain theoretical models, providing important clues on modes and rates of star formation in galaxies and on the importance of infall and outflows. $\mathrm{H}$ II regions indicate the present-day ( $10 \mathrm{Myr})$ gas-phase element abundances, while planetary nebulae (PNe) reveal the chemical composition of a galaxy at "intermediate" ages of a few 100 Myr to a few Gyr (or even up to 10 Gyr as for the case discussed below). These nebular abundances provide information on elements that are not easily observed in stellar absorptionline spectra. Ideally one wishes to combine information from nebular and stellar abundances, but nebular emission-line spectra have the added advantage of being observable at high signal to noise within a fraction of the telescope time required to obtain similarly deep stellar absorption-line data. For the Local Group and other nearby galaxies these abundance data can be combined with star formation histories derived from color-magnitude diagrams of resolved stars, thereby yielding deeper insights on galaxy evolution, in particular on the overall chemical evolution of galaxies as a function of time.

* Based on observations obtained at the European Southern Observatory, La Silla, Chile (program 072.A-0087(B)).
The Local Group consists of three spiral galaxies and a large number of dwarf galaxies including irregulars, ellipticals, and spheroidals (see, e.g., Grebel 2001, for a recent review). The least massive, least luminous, most gas-deficient and most numerous dwarf galaxies are the dwarf spheroidals (dSphs). Only in two dSph galaxies in the Local Group have PNe been detected to date: one in Fornax (Danziger et al. 1978) and four in Sagittarius (Zijlstra et al. 1996, 2006). Searches for PNe in other dSph galaxies yielded no detections. For example, no PNe were found with Sloan Digital Sky Survey (York et al. 2000; Stoughton et al. 2001) data for the Sextans, Draco and Leo I dSph galaxies (Kniazev et al. 2005b). The few PNe known are valuable in order to compare nebular abundances of gas-deficient galaxies like dSphs with those of gas-rich galaxies (e.g., dwarf irregulars). In fact, in gas-deficient galaxies without $\mathrm{H}$ II regions, PNe are the only means of obtaining nebular oxygen abundances (see, e.g., Richer et al. 1998). This is of particular importance when considering metallicity-luminosity relations of galaxies, since ideally one would like to use comparable metallicity indicators for these kinds of studies (see, e.g., the discussion in Grebel et al. 2003).

The goal of our present work is to improve our knowledge of the element abundances of the lone PN in the Fornax dSph through new high-quality New Technology Telescope (NTT) spectrophotometry. Fornax was discovered by Shapley (1938) and has a distance of about $140 \mathrm{kpc}$. With an absolute $V$-band 
magnitude of -13.1 it is one of the most luminous dSphs known (e.g., Table 1 in Grebel et al. 2003). It is also one of only two dSphs known to contain globular clusters. Several of its metalpoor globular clusters are as old as the oldest Galactic clusters (Buonanno et al. 1999), adding Fornax to the list of nearby galaxies that share a common epoch of early star formation (Grebel \& Gallagher 2004). Fornax contains a prominent old population, but most of its stars formed at intermediate ages (e.g., Stetson et al. 1998). Unlike other dSphs, Fornax continued to form stars until approximately $200 \mathrm{Myr}$ ago (Grebel \& Stetson 1999; Saviane et al. 2000). Its younger population is located in its central regions as seems generally to be the case in dSphs with extended star formation histories (e.g., Grebel 1999, 2000; Harbeck et al. 2001). However, in Fornax the young population shows a peculiarly asymmetric, almost $V$-shaped distribution (Stetson et al. 1998). Moreover, the centroids of the distributions of Fornax' older populations are shifted with respect to each other. Recent wide-field studies by Coleman et al. (2004) revealed two shell-like structures that may indicate a past merger event in Fornax. Dinescu et al. (2004) suggest that the termination of star formation in Fornax about 200 Myr ago may have been caused by a crossing of the Magellanic stream, which stripped Fornax of its star-forming material.

The metallicities of the stars in Fornax have been studied repeatedly using both photometric techniques and spectroscopy. The photometric studies revealed a considerable abundance spread with a relatively high mean metallicity (e.g., Grebel 1997; Saviane et al. 2000).

Several spectroscopic studies of Fornax have been carried out in recent years. For instance, Pont et al. (2004) measured metallicities for 117 red giants and found a surprisingly high mean metallicity of $[\mathrm{Fe} / \mathrm{H}]=-0.9$ dex with a substantial metalpoor tail. They also demonstrate that Fornax has a complex age-metallicity relation and that it underwent considerable enrichment over the past few Gyr. A recent high-resolution study of three red giants in Fornax was conducted by Tolstoy et al. (2003). This work illustrates that Fornax follows the trend of high (near-solar) $[\alpha / \mathrm{Fe}]$ ratios at low $[\mathrm{Fe} / \mathrm{H}]$ that was found in other dSphs, setting these stars apart from the ones in the Galactic halo (e.g., Shetrone et al. 2001). Battaglia et al. (2006) obtained metallicities for 562 stars in Fornax and found a wide range of abundances covering -2.8 to -0.1 dex in $[\mathrm{Fe} / \mathrm{H}]$. The more metal-poor component peaks at $-1.7 \mathrm{dex}$ and the more metal-rich one at -0.9 dex. Also, these authors find the more metal-rich and younger stars to be more centrally concentrated in good agreement with earlier studies cited above. While a wide range in metallicities is quite common even in $\mathrm{dSphs}$ with predominantly old populations (e.g., Shetrone et al. 2001), the range in stellar abundances in Fornax exceeds even that found in other dSphs with extended star formation histories (e.g., Koch et al. 2006, 2007) since Fornax formed stars over almost the entire Hubble time. Nonetheless it remains difficult to assign ages to individual red giants even if their metallicity is known.

The PN in the Fornax dSph galaxy, discovered by Danziger et al. (1978), remains the only one known in this dwarf. Danziger et al. obtained the first spectroscopic observations of it. Their results and those of others are discussed in Sect. 4.

The contents of this paper are organized as follows. Section 2 gives the description of our observations and of the data reduction. Our results are summarized in Sect. 3 and discussed Sect. 4. The conclusions drawn from this study are summarized in Sect. 5.

\section{Spectral observations and data reduction}

Our spectrophotometric observations were carried out with the NTT at the European Southern Observatory at La Silla on February 14, 2004. The observations were performed with the Red Arm of the EMMI multipurpose instrument using a long slit of $8^{\prime} \times 2^{\prime \prime}$. For these observations the CCD rows were binned by a factor of 2, yielding a final spatial sampling of $0.33^{\prime \prime}$ pixel $^{-1}$. The seeing during the observations was very stable and varied within the range of $0.4^{\prime \prime}$ to $0.6^{\prime \prime}$. The whole spectral range covered by the two grisms was $3800-8700 \AA$ with a sampling of $1.63 \AA$ pixel $^{-1}$ in the blue part (3800-7000 $\AA$; spectral resolution $(F W H M)$ of $\sim 7 \AA$ ) and with a sampling of $1.4 \AA$ pixel $^{-1}$ in the red part (5750-8670 $\AA$; spectral resolution of $\sim 6.5 \AA$ ). The PN position in Fornax was taken from Danziger et al. (1978). A one-minute $\mathrm{H} \alpha$ acquisition image was obtained before the spectroscopic observations in order to select an optimal position of the slit. The total exposure times were $40 \mathrm{~min}$ for each grism. The exposures were broken up into 2 sub-exposures with 20 min of exposure time each to facilitate the removal of cosmic rays. The airmass changed from 1.3 for the first sub-exposure to 1.75 for the last sub-exposure. Spectra of He-Ar comparison arcs were obtained to calibrate the wavelength scale. The spectrophotometric standard stars GD 108, GD 50, and G 60-54 were observed with a $10^{\prime \prime}$ slit width at the beginning, middle, and end of the night for flux calibration.

The reduction of all data was performed using the standard data reduction packages MIDAS ${ }^{1}$ and IRAF $^{2}$. Cosmic ray hits were removed from the $2 \mathrm{D}$ spectral frame in MIDAS. Using IRAF tasks in the ccdred package, we subtracted the bias and performed flat-field corrections. After that the $2 \mathrm{D}$ spectrum was wavelength-calibrated and the night sky background was subtracted. Using our data of the spectrophotometry standard stars, the intensities of the 2D spectrum were transformed to absolute fluxes. One-dimensional spectra were extracted such as to get total emission line fluxes. All sensitivity curves observed during the night were compared. We found the final curves to have a precision better than $2 \%$ over the whole optical range, except for the region blueward of $4000 \AA$ where the sensitivity drops off rapidly.

All emission lines were measured applying the MIDAS programs described in Kniazev et al. (2000, 2004): These programs determine the location of the continuum, perform a robust noise estimation, and fit separate lines by a single Gaussian superimposed on the continuum-subtracted spectrum. Some overlapping lines were fitted simultaneously as a blend of two or more Gaussian features: the $\mathrm{H} \alpha \lambda 6563$ and [N II] $\lambda \lambda 6548,6584$ lines, the [S II] $\lambda \lambda 6716,6731$ lines, and the [O II] $\lambda \lambda 7320,7330$ lines. The emission lines He I $\lambda 5876$, [O I] $\lambda 6300$, [S III] $\lambda 6312$, [O I] $\lambda 6364, \mathrm{H} \alpha \lambda 6563$, [N II] $\lambda \lambda 6548,6584$, He I $\lambda 6678$, and [S II] $\lambda \lambda 6716,6731$ were detected independently in both grism spectra. Their intensities were averaged after applying weights inversely proportional to the errors of the measurements. The quoted errors of single line intensities include some components that are summed up in quadrature. The total errors were propagated in the calculations and are included in the uncertainties

1 MIDAS is an acronym for the European Southern Observatory package - Munich Image Data Analysis System.

2 IRAF is distributed by National Optical Astronomical Observatories, which is operated by the Association of Universities for Research in Astronomy, Inc., under cooperative agreement with the National Science Foundation. 
Table 1. Line intensities in the observed spectra of the Fornax PN.

\begin{tabular}{|c|c|c|}
\hline$\lambda_{0}(\AA)$ Ion & $F(\lambda) / F(\mathrm{H} \beta)$ & $I(\lambda) / I(\mathrm{H} \beta)$ \\
\hline $3868[\mathrm{Ne}$ III] & $0.2428 \pm 0.0149$ & $0.2434 \pm 0.0154$ \\
\hline $3889 \mathrm{He} \mathrm{I}+\mathrm{H} 8$ & $0.2028 \pm 0.0072$ & $0.2269 \pm 0.0101$ \\
\hline $3967[\mathrm{Ne}$ III $]+\mathrm{H} 7$ & $0.2441 \pm 0.0076$ & $0.2697 \pm 0.0107$ \\
\hline $4026 \mathrm{He} \mathrm{I}$ & $0.0208 \pm 0.0019$ & $0.0208 \pm 0.0019$ \\
\hline $4101 \mathrm{H} \delta$ & $0.2400 \pm 0.0072$ & $0.2624 \pm 0.0098$ \\
\hline 4267 C II & $0.0102 \pm 0.0012$ & $0.0102 \pm 0.0012$ \\
\hline $4340 \mathrm{H} \gamma$ & $0.4464 \pm 0.0129$ & $0.4627 \pm 0.0145$ \\
\hline 4363 [O III] & $0.0371 \pm 0.0016$ & $0.0369 \pm 0.0016$ \\
\hline $4388 \mathrm{He} \mathrm{I}$ & $0.0057 \pm 0.0009$ & $0.0056 \pm 0.0009$ \\
\hline $4471 \mathrm{He} \mathrm{I}$ & $0.0488 \pm 0.0019$ & $0.0484 \pm 0.0019$ \\
\hline $4658[\mathrm{Fe} \mathrm{III}]$ & $0.0032 \pm 0.0013$ & $0.0032 \pm 0.0013$ \\
\hline $4686 \mathrm{He}$ II & $0.0028 \pm 0.0009$ & $0.0028 \pm 0.0009$ \\
\hline $4713[\mathrm{Ar}$ IV $]+\mathrm{He} \mathrm{I}$ & $0.0082 \pm 0.0010$ & $0.0081 \pm 0.0010$ \\
\hline 4740 [Ar IV] & $0.0008 \pm 0.0004$ & $0.0007 \pm 0.0004$ \\
\hline $4861 \mathrm{H} \beta$ & $1.0000 \pm 0.0286$ & $1.0000 \pm 0.0291$ \\
\hline $4922 \mathrm{He} \mathrm{I}$ & $0.0141 \pm 0.0010$ & $0.0139 \pm 0.0010$ \\
\hline 4959 [O III] & $1.8359 \pm 0.0524$ & $1.8101 \pm 0.0523$ \\
\hline 5007 [O III] & $5.4626 \pm 0.1555$ & $5.3824 \pm 0.1554$ \\
\hline $5048 \mathrm{He} \mathrm{I}$ & $0.0030 \pm 0.0012$ & $0.0030 \pm 0.0012$ \\
\hline 5538 [Cl III] & $0.0013 \pm 0.0007$ & $0.0013 \pm 0.0007$ \\
\hline $5876 \mathrm{He} \mathrm{I}$ & $0.1464 \pm 0.0043$ & $0.1428 \pm 0.0044$ \\
\hline $6312[\mathrm{~S}$ III] & $0.0054 \pm 0.0006$ & $0.0052 \pm 0.0006$ \\
\hline $6548[\mathrm{~N} \mathrm{III}]$ & $0.0194 \pm 0.0009$ & $0.0188 \pm 0.0009$ \\
\hline $6563 \mathrm{H} \alpha$ & $2.9620 \pm 0.0852$ & $2.8745 \pm 0.0909$ \\
\hline 6584 [N II] & $0.0578 \pm 0.0034$ & $0.0560 \pm 0.0034$ \\
\hline $6678 \mathrm{He} \mathrm{I}$ & $0.0408 \pm 0.0015$ & $0.0395 \pm 0.0015$ \\
\hline 6717 [S II] & $0.0044 \pm 0.0007$ & $0.0043 \pm 0.0007$ \\
\hline $6731[\mathrm{~S}$ II] & $0.0047 \pm 0.0007$ & $0.0045 \pm 0.0007$ \\
\hline $7065 \mathrm{He} \mathrm{I}$ & $0.0538 \pm 0.0019$ & $0.0519 \pm 0.0020$ \\
\hline $7136[\mathrm{Ar}$ III] & $0.0596 \pm 0.0021$ & $0.0574 \pm 0.0022$ \\
\hline 7236 [Ar IV] & $0.0148 \pm 0.0012$ & $0.0142 \pm 0.0012$ \\
\hline $7281 \mathrm{He} \mathrm{I}$ & $0.0083 \pm 0.0009$ & $0.0080 \pm 0.0008$ \\
\hline 7320 [O II] & $0.0089 \pm 0.0009$ & $0.0086 \pm 0.0009$ \\
\hline $7330[\mathrm{O}$ II] & $0.0085 \pm 0.0011$ & $0.0082 \pm 0.0011$ \\
\hline 7751 [Ar III] & $0.0145 \pm 0.0011$ & $0.0140 \pm 0.0011$ \\
\hline 8438 P18 & $0.0022 \pm 0.0006$ & $0.0021 \pm 0.0006$ \\
\hline 8467 P17 & $0.0027 \pm 0.0006$ & $0.0025 \pm 0.0006$ \\
\hline 8502 P16 & $0.0039 \pm 0.0007$ & $0.0038 \pm 0.0007$ \\
\hline 8545 P15 & $0.0080 \pm 0.0009$ & $0.0076 \pm 0.0009$ \\
\hline 8598 P14 & $0.0058 \pm 0.0009$ & $0.0056 \pm 0.0009$ \\
\hline 8665 P13 & $0.0080 \pm 0.0009$ & $0.0077 \pm 0.0009$ \\
\hline$C(\mathrm{H} \beta) \operatorname{dex}$ & \multicolumn{2}{|c|}{$0.03 \pm 0.01$} \\
\hline$F(\mathrm{H} \beta)^{a}$ & \multicolumn{2}{|c|}{$160 \pm 3$} \\
\hline Rad. vel. $\mathrm{km} \mathrm{s}^{-1}$ & \multicolumn{2}{|c|}{$72 \pm 14$} \\
\hline
\end{tabular}

${ }^{a}$ Observed flux in units of $10^{-16} \mathrm{erg} \mathrm{s}^{-1} \mathrm{~cm}^{-2}$.

of the element abundances and all derived parameters presented here.

\section{Results}

The relative intensities of all detected emission lines as well as the derived $\mathrm{C}(\mathrm{H} \beta)$, the measured flux in the $\mathrm{H} \beta$ emission line, and the measured heliocentric radial velocity are given in Table 1. The final 1D spectra are shown in Fig. 1. The electron temperature $T_{\mathrm{e}}$, the number density $N_{\mathrm{e}}$, and the ionic and total element abundances for $\mathrm{O}, \mathrm{N}, \mathrm{S}, \mathrm{Ne}, \mathrm{Cl}, \mathrm{Ar}$, and He were calculated in the same manner as detailed in Kniazev et al. (2005a). They are presented in Table 2. The [O II] $\lambda 3727,3729$ doublet was not observed, and $\mathrm{O}^{+} / \mathrm{H}^{+}$was calculated using the intensities of the [O II] $\lambda 7320,7330$ lines. The contribution to the intensities of the [O II] $\lambda 7320,7330$ lines due to recombination was taken into account following the correction from Liu et al. (2000). The electron temperatures for different ions were calculated following Garnett (1992) or Stasińska (1990) in the manner described in e.g., Izotov \& Thuan (1998). The ionization correction factors $\mathrm{ICF}(\mathrm{A})$ for different elements were calculated using the equations from Torres-Peimbert \& Peimbert (1977), Garnett (1990), and Izotov \& Thuan (1998) and references therein. The latter are derived for H II regions, but for weak He II there are only minor differences with the PNe ICFs of Kingsburgh \& Barlow (1994). Some Paschen lines are detected in the red part of the spectrum. The C II $\lambda 4267$ recombination line is also detected in our spectrum. The ionic abundance of $\mathrm{C}$ was derived using the calculations of Davey et al. (2000) for the case B effective recombination coefficients, which include both radiative and dielectronic recombination processes. Ionic abundances derived from optical recombination lines (ORLs) depend only weakly on the adopted temperature and are essentially independent of $N_{\mathrm{e}}$. A temperature of $T_{\mathrm{e}}=10500 \pm 500 \mathrm{~K}$ was assumed throughout. The ICF(A) for $\mathrm{C}$ was calculated using Kingsburgh \& Barlow (1994). The ionic and total element abundance for C are presented in Table 3.

After accounting for the Milky Way foreground extinction $A_{\mathrm{B}}=0.08-0 . \mathrm{m} 10$ in the direction of the Fornax dSph (Schlegel et al. 1998) we do not see any additional internal extinction for the $\mathrm{PN}$ or in the Fornax dSph at this position. Our observed $\mathrm{H} \beta$ flux of the PN is about the same as was measured by Danziger et al. (1978), who found $m_{5007}=18$ m 91 (Jacoby 1989). Our heliocentric radial velocity $V_{\text {hel }}=72 \pm 14$ is far from the value of $V_{\text {hel }}=10 \pm 40 \mathrm{~km} \mathrm{~s}^{-1}$ from Danziger et al. (1978), but within the uncertainties it is consistent with the Fornax optical velocity of $53 \pm 3 \mathrm{~km} \mathrm{~s}^{-1}$ (Mateo 1998; see also Walker et al. 2006; and Battaglia et al. 2006) and with the velocities of the Fornax globular clusters (Mateo et al. 1991 and references therein). Battaglia et al. (2006) find a line-of-sight $3 \sigma$ velocity dispersion of $13.7 \pm 0.4 \mathrm{~km} \mathrm{~s}^{-1}$ for Fornax' field stars.

Some of the physical parameters of the PN in Fornax can be estimated in the same way it was done by Kniazev et al. (2005a) for the PNe in Sextans A and Sextans B and by Kniazev et al. (2005b) for the PN in Leo A. With the He II $\lambda 4686$ and $\mathrm{H} \beta$ emission lines the effective temperature ( $\left.T_{\text {eff }}\right)$ of the PN central star can be estimated using the relation from Kaler \& Jacoby (1989). The weakness of the line returns the minimum temperature of this relation, which must be considered an upper limit. Also, the relation assumes optical thickness of the nebula which may be in doubt. The (He II $\lambda 4686) /(\mathrm{He}$ I 25876$)$ line ratios and (He II $\lambda 4686) /(\mathrm{He}$ I 24471$)$ can be reproduced only with cool central stars $\left(T_{\text {eff }} \sim 50-60 \mathrm{kK}\right)$ and optical depth $\tau_{13.6}<10$, according to the models of Gruenwald \& Viegas (2000). We therefore adopt $T_{\text {eff }}=55 \mathrm{kK}$.

The total luminosity $\left(L / L_{\odot}\right)$ can be derived from Zijlstra \& Pottasch (1989). This assumes an optically thick nebula. For the adopted $T_{\text {eff }}$, we find $L=1.0 \times 10^{3} L_{\odot}$, which is low for a cool PN central star. This is for a black-body model: a stellar atmosphere model may give a somewhat higher luminosity.

We have also derived the luminosity using the effective temperature and stellar magnitude. The latter is obtained from the continuum emission in the spectrum: at $5500 \AA$, we find $V=$ $20.13 \pm 0.05$. The absolute magnitude is $M_{V}=-0.60$. Using the non-LTE models of Rauch (2003), we find that for $T_{\text {eff }}=$ $50 \mathrm{kK}$ this corresponds to $L=6500 L_{\odot}$ and for $T_{\mathrm{eff}}=60 \mathrm{kK}$, $L=11300 L_{\odot}$. The difference with the luminosity derived above suggests that the nebula is not optically thick in all directions.

We finally can derive limits on the luminosity from dynamical considerations. The density derived above, together with the 

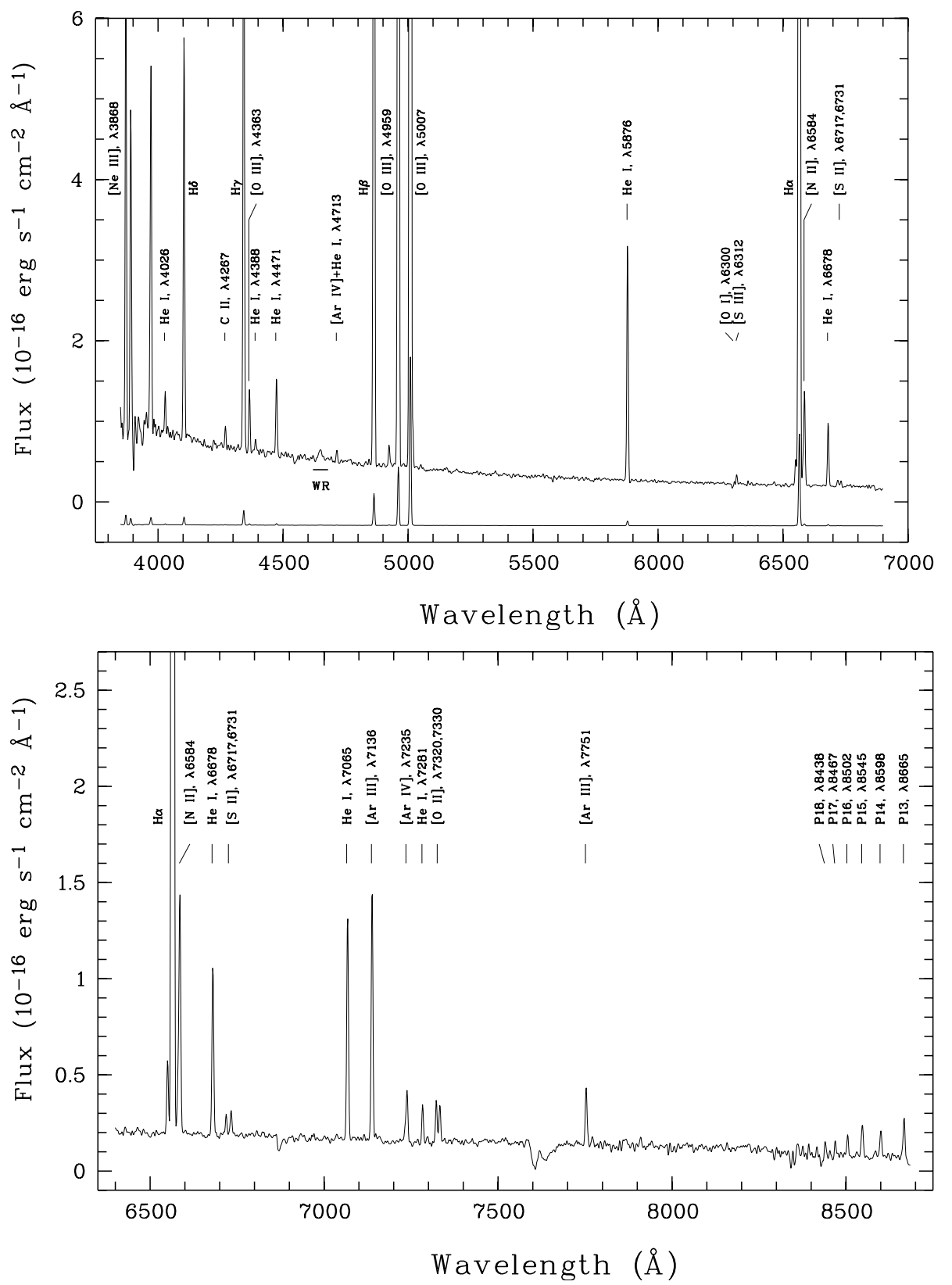

Fig. 1. One-dimensional reduced spectra of the planetary nebula in Fornax that were obtained with two grisms. The spectra cover a wavelength range of 3800-7000 A (top panel, grism \#5) and 6400-8650 Å (bottom panel, grism \#6). Most of the detected emission lines are marked. The spectrum at the bottom of the top panel is scaled by $1 / 40$ and shifted to show the relative intensities of the strong lines.

$\mathrm{H} \beta$ flux indicates a mass of $0.33 M_{\odot}$ and a radius of $0.16 \mathrm{pc}$ (assuming constant density). (The radius of the nebula has however not been directly confirmed.) For an expansion velocity of $20 \mathrm{~km} \mathrm{~s}^{-1}$ (Richer 2005), this implies an age of the ejecta of roughly $8000 \mathrm{yr}$. The models of stellar temperature versus dynamical age (Gesicki et al. 2006) show that $T_{\text {eff }}=55 \mathrm{kK}$ is reached after this time for models with a stellar mass of $0.58 M_{\odot}$. The luminosity of such a star is $L=5000 L_{\odot}$.

All three derivations have uncertainties. Combining the last two, we adopt $L=7000 L_{\odot}$.

After that the mass of the progenitor is obtained using the theoretical tracks of Vassiliadis \& Wood (1994). According to Sect. 4 the metallicity is $Z \sim 1 / 4 Z_{\odot}$ for the PN in Fornax, where the solar value of the oxygen abundance is $12+\log (\mathrm{O} / \mathrm{H})=8.66$ (Asplund et al. 2004). Hence the tracks for $Z=0.004\left(1 / 5\right.$ of $Z_{\odot}$ ) were chosen, which are the tracks closest to the above metallicity. The approximate age of the PN progenitor $\left(t_{\mathrm{MS}}\right)$ is derived using the evolutionary lifetimes of various phases of stars from Vassiliadis \& Wood (1993). All these derived parameters are summarized in Table 4 . In addition, $m_{5007}$ (Jacoby 1989) and A 5007 (Cardelli et al. 1989) were calculated.

\section{Discussion}

\subsection{Element abundances of the PN in Fornax}

Danziger et al. (1978) carried out the first spectroscopic observations of the PN in the Fornax dSph galaxy. They measured the [O III] $\lambda 4363$ line with an accuracy $\sim 30 \%$ and calculated $12+\log (\mathrm{O} / \mathrm{H})=8.51$ as well as $\mathrm{He}, \mathrm{Ar}$, and $\mathrm{N}$ abundances. Their data permitted them only to present an upper limit for the [S II] $\lambda \lambda 6717,6731$ lines. Maran et al. (1984) recalculated the oxygen abundance $(12+\log (\mathrm{O} / \mathrm{H})=8.38)$ as well as the abundances of $\mathrm{He}, \mathrm{Ar}, \mathrm{N}$, and $\mathrm{S}$ based on line intensities from Danziger et al. (1978). Walsh et al. (1997) tried also to observe the spectrum of the PN in Fornax, but they did not detect the [O III] $\lambda 4363$ line nor the [S II] $\lambda \lambda 6717,6731$ lines. Richer \& McCall (1995) estimated an oxygen abundance of the PN in Fornax that is 0.4 dex lower than the one calculated by 
Table 2. Physical parameters and $\mathrm{O}, \mathrm{N}, \mathrm{Ne}, \mathrm{S}, \mathrm{Ar}, \mathrm{Cl}, \mathrm{Fe}$ and $\mathrm{He}$ Abundances of the PN in Fornax.

\begin{tabular}{|c|c|}
\hline Parameter & $\overline{\text { Value }}$ \\
\hline $\bar{T} T_{\mathrm{e}}(\mathrm{OIII})(\mathrm{K})$ & $10200 \pm 160$ \\
\hline$T_{\mathrm{e}}(\mathrm{OII})(\mathrm{K})$ & $11050 \pm 150$ \\
\hline$T_{\mathrm{e}}(\mathrm{ArIII})(\mathrm{K})$ & $10650 \pm 130$ \\
\hline$T_{\mathrm{e}}(\mathrm{SIII})(\mathrm{K})$ & $10650 \pm 130$ \\
\hline$T_{\mathrm{e}}(\mathrm{ClIII})(\mathrm{K})$ & $10650 \pm 130$ \\
\hline$N_{\mathrm{e}}(\mathrm{SII})\left(\mathrm{cm}^{-3}\right)$ & $750 \pm 270$ \\
\hline $\mathrm{O}^{+} / \mathrm{H}^{+}\left(\times 10^{5}\right)$ & $1.411 \pm 0.121$ \\
\hline $\mathrm{O}^{++} / \mathrm{H}^{+}\left(\times 10^{5}\right)$ & $17.750 \pm 0.999$ \\
\hline $\mathrm{O} / \mathrm{H}\left(\times 10^{5}\right)$ & $19.160 \pm 1.006$ \\
\hline $12+\log (\mathrm{O} / \mathrm{H})$ & $8.28 \pm 0.02$ \\
\hline $\mathrm{N}^{+} / \mathrm{H}^{+}\left(\times 10^{7}\right)$ & $8.020 \pm 0.452$ \\
\hline $\mathrm{ICF}(\mathrm{N})$ & 13.577 \\
\hline $\mathrm{N} / \mathrm{H}\left(\times 10^{5}\right)$ & $1.09 \pm 0.06$ \\
\hline $12+\log (\mathrm{N} / \mathrm{H})$ & $7.04 \pm 0.02$ \\
\hline $\log (\mathrm{N} / \mathrm{O})$ & $-1.25 \pm 0.03$ \\
\hline $\mathrm{Ne}^{++} / \mathrm{H}^{+}\left(\times 10^{5}\right)$ & $2.198 \pm 0.189$ \\
\hline $\mathrm{ICF}(\mathrm{Ne})$ & 1.079 \\
\hline $\mathrm{Ne} / \mathrm{H}\left(\times 10^{5}\right)$ & $2.37 \pm 0.20$ \\
\hline $12+\log (\mathrm{Ne} / \mathrm{H})$ & $7.38 \pm 0.04$ \\
\hline $\log (\mathrm{Ne} / \mathrm{O})$ & $-0.91 \pm 0.04$ \\
\hline $\mathrm{S}^{+} / \mathrm{H}^{+}\left(\times 10^{7}\right)$ & $0.172 \pm 0.024$ \\
\hline $\mathrm{S}^{++} / \mathrm{H}^{+}\left(\times 10^{7}\right)$ & $9.033 \pm 1.129$ \\
\hline $\mathrm{ICF}(\mathrm{S})$ & 3.075 \\
\hline $\mathrm{S} / \mathrm{H}\left(\times 10^{7}\right)$ & $28.31 \pm 3.47$ \\
\hline $12+\log (\mathrm{S} / \mathrm{H})$ & $6.45 \pm 0.05$ \\
\hline $\log (\mathrm{S} / \mathrm{O})$ & $-1.83 \pm 0.06$ \\
\hline $\mathrm{Ar}^{++} / \mathrm{H}^{+}\left(\times 10^{7}\right)$ & $4.099 \pm 0.198$ \\
\hline $\mathrm{Ar}^{+++} / \mathrm{H}^{+}\left(\times 10^{7}\right)$ & $0.315 \pm 0.161$ \\
\hline $\mathrm{ICF}(\mathrm{Ar})$ & 1.006 \\
\hline $\mathrm{Ar} / \mathrm{H}\left(\times 10^{7}\right)$ & $4.44 \pm 0.26$ \\
\hline $12+\log (\mathrm{Ar} / \mathrm{H})$ & $5.65 \pm 0.03$ \\
\hline $\log (\mathrm{Ar} / \mathrm{O})$ & $-2.63 \pm 0.03$ \\
\hline $\mathrm{Cl}^{++} / \mathrm{H}^{+}\left(\times 10^{7}\right)$ & $0.154 \pm 0.055$ \\
\hline $\mathrm{ICF}(\mathrm{Cl})$ & 2.599 \\
\hline $\mathrm{Cl} / \mathrm{H}\left(\times 10^{7}\right)$ & $0.40 \pm 0.14$ \\
\hline $12+\log (\mathrm{Cl} / \mathrm{H})$ & $4.60 \pm 0.15$ \\
\hline $\log (\mathrm{Cl} / \mathrm{O})$ & $-3.68 \pm 0.16$ \\
\hline $\mathrm{Fe}^{++} / \mathrm{H}^{+}\left(\times 10^{7}\right)$ & $1.412 \pm 0.582$ \\
\hline $\mathrm{ICF}(\mathrm{Fe})$ & 16.971 \\
\hline $\log (\mathrm{Fe} / \mathrm{O})$ & $-1.90 \pm 0.18$ \\
\hline [O/Fe] & $0.48 \pm 0.18$ \\
\hline$[\mathrm{Fe} / \mathrm{H}]$ & $-1.13 \pm 0.18$ \\
\hline $\mathrm{He}^{+} / \mathrm{H}^{+}$ & $0.0922 \pm 0.0020$ \\
\hline $\mathrm{He}^{++} / \mathrm{H}^{+}$ & $0.0002 \pm 0.0001$ \\
\hline $\mathrm{He} / \mathrm{H}$ & $0.0924 \pm 0.0020$ \\
\hline $12+\log (\mathrm{He} / \mathrm{H})$ & $10.97 \pm 0.01$ \\
\hline
\end{tabular}

Table 3. $\mathrm{C}$ abundance of the PN in Fornax.

\begin{tabular}{lc}
\hline \hline Parameter & Value \\
\hline $\mathrm{C}^{++} / \mathrm{H}^{+}\left(\times 10^{4}\right)$ & $9.82 \pm 1.44$ \\
$\mathrm{ICF}(\mathrm{C})$ & 1.08 \\
$\mathrm{C} / \mathrm{H}\left(\times 10^{3}\right)$ & $1.06 \pm 0.16$ \\
$12+\log (\mathrm{C} / \mathrm{H})$ & $9.02 \pm 0.07$ \\
$\log (\mathrm{C} / \mathrm{O})$ & $0.74 \pm 0.09$ \\
\hline
\end{tabular}

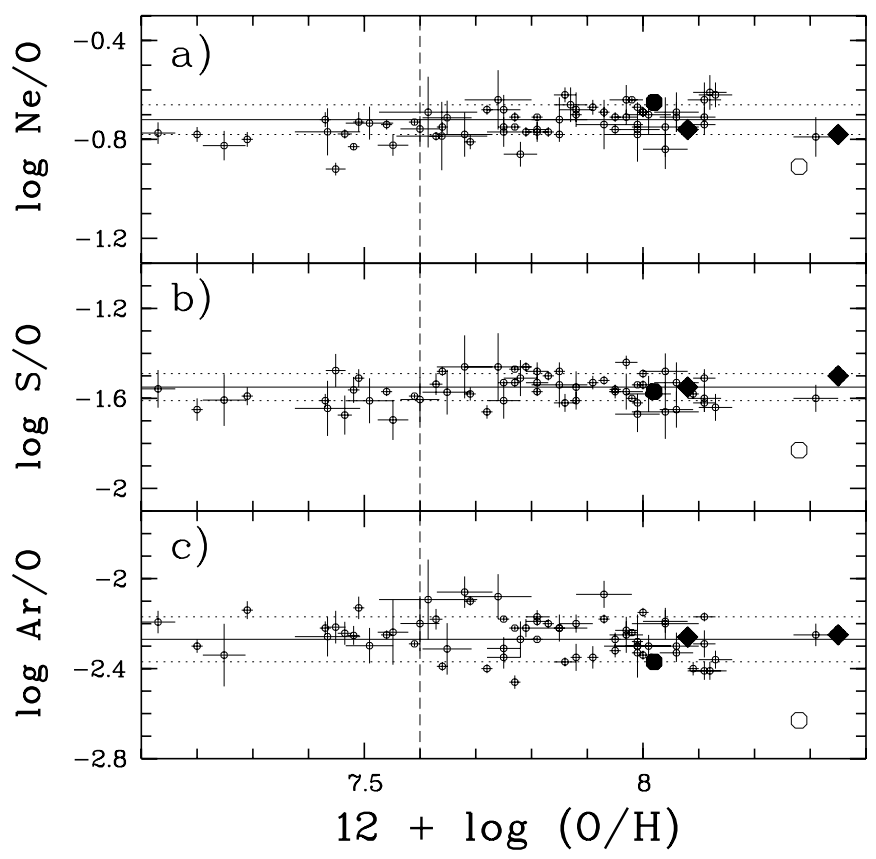

Fig. 2. $\alpha$-element-to-oxygen abundance ratios for $\log (\mathrm{Ne} / \mathrm{O}), \log (\mathrm{S} / \mathrm{O})$ and $\log (\mathrm{Ar} / \mathrm{O})$ with their $1 \sigma$ errors (short-dashed lines) from Izotov \& Thuan (1999) as a function of oxygen abundance. The long-dashed line shows the value $12+\log (\mathrm{O} / \mathrm{H})=7.60$ above which these abundance ratios were calculated. Data for H II regions from Izotov \& Thuan (1999) and from Kniazev et al. (2003) are overplotted. Our calculated ratios for the PN in Fornax are shown as open circles. The same ratios corrected for self-pollution in oxygen are shown as filled circles. For comparison data for the two of the PNe in the Sagittarius dSph galaxy (Dudziak et al. 2000) are shown as filled lozenges. The chemical abundances for Sagittarius' PNe were recalculated with the same method based on published spectral data.

Table 4. Physical parameters of the Fornax PN and its progenitor.

\begin{tabular}{lc}
\hline \hline Parameter & Value \\
\hline$T_{\text {eff }}(\mathrm{K})$ & 55000 \\
$\log 10\left(T_{\text {eff }}\right)$ & 4.74 \\
$L / L_{\odot}$ & 7000 \\
$\log \left(L / L_{\odot}\right)$ & 3.85 \\
$M / M_{\odot}$ & $\sim 1.2$ \\
$t_{\mathrm{MS}}(\mathrm{Gyr})$ & $\sim 7.0$ \\
& \\
$m_{5007}$ & $19.01 \pm 0^{\mathrm{m}} 02$ \\
$A_{5007}$ & 0.07 \\
$V_{*}(\mathrm{mag})$ & $20^{\mathrm{m}} \cdot 13 \pm 0.05$ \\
\hline
\end{tabular}

Maran et al. (1984). These authors exploit this correction by using the fact that the oxygen abundance can be slightly higher for the brightest PN of a galaxy. However, Walsh et al. (1997) argued that if a galaxy has only one PN, then such a correction is not applicable.

Our data are considerably better (see Fig. 1) than the earlier spectra, yielding a signal-to-noise ratio of 22 for the [O III] $\lambda 4363$ line. With our new data we have for the first time detected the weak [S II] $\lambda \lambda 6717,6731$ lines $(I(6717+6731) \approx 0.01 I(\mathrm{H} \beta))$ and determined the electron number density. We obtain an oxygen abundance of $12+\log (\mathrm{O} / \mathrm{H})=$ 8.28 for the Fornax PN.

In $\mathrm{H}$ II regions, the oxygen that is seen has been produced by the same massive stars that produced the alpha-process elements 


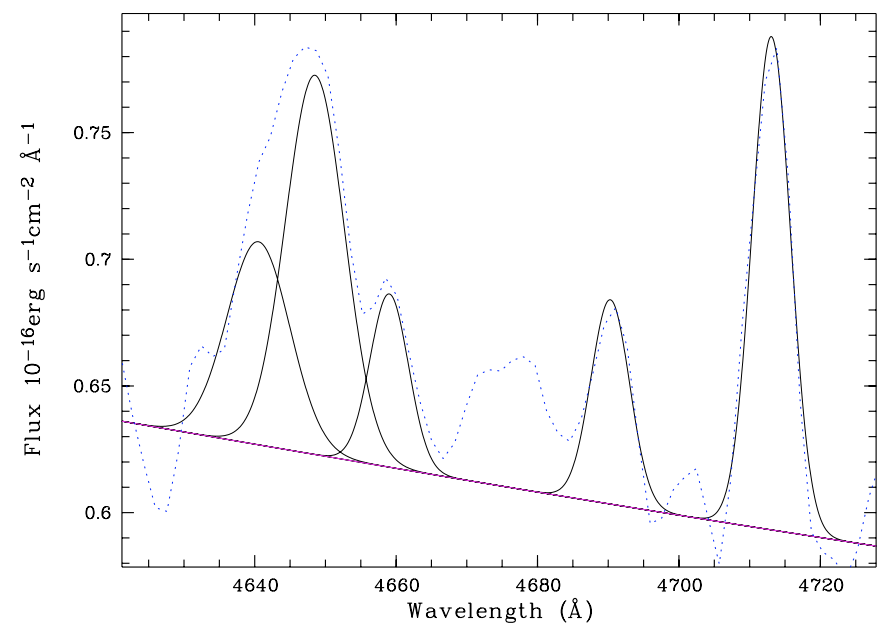

Fig. 3. Gaussian multicomponent fitting of the blue Wolf-Rayet bump. The observed spectrum in the fitted region and the continuum level are shown. The following lines from left to right are marked: N III $\lambda 4640$,

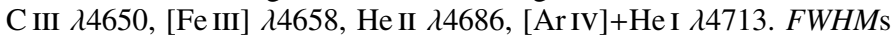
for the [Fe III] $\lambda 4658$, He II $\lambda 4686$ and [Ar IV]+He I $\lambda 4713$ lines are similar within the uncertainties.

neon, sulfur and argon. Therefore $\log (\mathrm{Ne} / \mathrm{O}), \log (\mathrm{S} / \mathrm{O})$, and $\log (\mathrm{Ar} / \mathrm{O})$ should be constant and show no dependence on the oxygen abundance. These $\alpha$-element-to-oxygen abundance ratios were measured by Izotov \& Thuan (1999) in H II regions in blue compact galaxies and found to be $\log (\mathrm{Ne} / \mathrm{O})=-0.72 \pm 0.06$, $\log (\mathrm{S} / \mathrm{O})=-1.55 \pm 0.06$ and $\log (\mathrm{Ar} / \mathrm{O})=-2.27 \pm 0.10$ for $12+\log (\mathrm{O} / \mathrm{H})>7.60$ as shown in Fig. 2. For H II regions the $\log (\mathrm{Cl} / \mathrm{O})$ ratio also does not show any significant increase with increasing oxygen abundance (Guseva et al. 2003; Esteban et al. 1998, 1999).

In contrast to $\mathrm{H}$ II regions some elemental abundances in PNe are affected by the nucleosynthesis in the PN progenitors. Newly synthesized material can be dredged up by convection in the envelope, significantly altering abundances of $\mathrm{He}, \mathrm{C}$, and $\mathrm{N}$ in the surface layers during the evolution of the PN progenitor stars on the giant branch and asymptotic giant branch (AGB). But also a certain amount of $\mathrm{O}$ can be mixed in during the thermally pulsing phase of AGB evolution. For example, Kingsburgh \& Barlow (1994) found that the $O$ abundance is altered by $\sim 0.2$ dex by AGB evolution. Kniazev et al. (2005a) studied Type I PNe in Sextans A and found significant self-pollution of the PN progenitor by a factor of $\sim 10$ in oxygen. Pequignot et al. (2000) show that the third dredge-up increases the $\mathrm{O}$-abundance at low metallicity but decreases it at high metallicity, due to the specific abundance ratios in the dredge-up material. Marigo (2001) also found this in theoretical dredge-up calculations.

Our new measurements of $\mathrm{O}, \mathrm{Ne}, \mathrm{Ar}$, and $\mathrm{S}$ abundances for the $\mathrm{PN}$ in Fornax are shown in Fig. 2 as empty circles. All $\log (\mathrm{Ne} / \mathrm{O}), \log (\mathrm{S} / \mathrm{O})$, and $\log (\mathrm{Ar} / \mathrm{O})$ ratios for this $\mathrm{PN}$ are systematically below the values that were determined for H II regions. This can be easily explained by additional enrichment in oxygen in the progenitor of the Fornax PN. Using both the abundance ratios of Izotov \& Thuan (1999) and our observed ratios this self-pollution can be calculated as weighted average $\delta \mathrm{O}=$ $0.27 \pm 0.10 \mathrm{dex}$. After correction the resulting oxygen abundance $12+\log (\mathrm{O} / \mathrm{H})$ is 8.01 dex. The corrected ratios $\left(\log (\mathrm{Ne} / \mathrm{O})_{\text {corr }}=\right.$ $-0.64, \log (\mathrm{S} / \mathrm{O})_{\text {corr }}=-1.56$ and $\left.\log (\mathrm{Ar} / \mathrm{O})_{\text {corr }}=-2.36\right)$ are consistent with the values in $\mathrm{H}$ II regions, showing that a change in $\mathrm{O}$ suffices. The corrected ratios are shown in Fig. 2 as filled circles. The chemical abundances for two of the PNe in the Sagittarius dSph galaxy were recalculated with the same method on the base of published spectral data. Our figure shows that the abundances of both PNe in Sagittarius are located in the area where they are expected to lie. We note here that according to our calculations these $\mathrm{PNe}$ have different oxygen abundances. Dudziak et al. (2000) find from detailed photoionization modeling that their abundances are very similar. The Sgr PN Wray 16423 has $12+\log (\mathrm{O} / \mathrm{H})=8.35 \pm 0.02$ according to our calculations, a value that is very close to the previously published $8.33 \pm 0.02$. But the Sgr PN He 2-436 has $12+\log (\mathrm{O} / \mathrm{H})=8.08 \pm 0.02$, which differs from the earlier result of $8.36 \pm 0.06$ (Dudziak et al. 2000).

Our abundance for the PN in Fornax reflects the ISM metallicity at the epoch of the PN progenitor formation about $8 \mathrm{Gyr}$ ago (see Table 4). Star formation histories for Fornax based on studies of its stellar populations (e.g., Grebel 1999; Tolstoy et al. 2003) indicate that the PN progenitor in Fornax originated at the time the star formation rate in Fornax reached its peak. Our $[\mathrm{Fe} / \mathrm{H}]$ result is based on the faint line [Fe III] $\lambda 4658$ we derived as the result of WR bump fitting; it may represent an upper limit. Using the fitted line strength yields $[\mathrm{Fe} / \mathrm{H}]=-1.13 \pm 0.18$ for the Fornax PN. This is very close to the photometrically derived mean metallicity of Fornax's old population -1.2 dex (Grebel et al. 2003) and well within the range of metallicities derived from spectroscopic measurements of red giant stars (Ca triplet) at that epoch (see Fig. 23 in Battaglia et al. 2006), although somewhat at the metal-rich part of that distribution. The $\alpha$ elements give a slightly higher abundance of $[\mathrm{O} / \mathrm{H}] \sim-0.7$. At $[\mathrm{Fe} / \mathrm{H}]=-1$, chemical evolution models give $[\mathrm{O} / \mathrm{Fe}]$ for dwarf spheroidal galaxies in the range $0-+0.5$ (Lanfranchi \& Matteucci 2004), declining with increasing $[\mathrm{Fe} / \mathrm{H}]$ : this is consistent with our value of $[\mathrm{O} / \mathrm{Fe}] \approx 0.5$. We note some caveats on this apparent agreement. First, the models do not fit the Sgr dSph galaxy well, where the stars show a $[\mathrm{O} / \mathrm{Fe}] \approx 0$ even at low metallicity. Second, our value is based on an indirect line identification which requires confirmation: if an upper limit, a lower Fe abundance is derived. Third, $\mathrm{Fe}$ is strongly depleted in many $\mathrm{PNe}$ (e.g., Rodríguez \& Rubin 2005), either due to dust condensation or due to iron processing during the s-process nucleosynthesis. Although at face value we find no evidence for depletion, it can not be ruled out either.

Our $[\mathrm{Fe} / \mathrm{H}]$ or $[\mathrm{O} / \mathrm{H}]$ value and $\mathrm{PN}$ progenitor age are consistent with the (relatively flat) age-metallicity relation for the Fornax dSph presented by Tolstoy et al. (2003), by Pont et al. (2004), and by Battaglia et al. (2006). The PN predates the sudden steep rise in metallicity seen for the youngest Fornax stars and for the youngest Sgr PN. The young, metal-rich population present in Fornax and Sgr may show the effect of accretion of enriched gas from the Milky Way (Zijlstra et al. 2006) or from the proposed merger event (Coleman et al. 2004).

The dichotomy between the ORL and CEL (collisionally excited lines) abundances is an open problem in nebular astrophysics (see Liu 2003, and references therein). Heavy-element abundances derived from ORLs are systematically higher than those derived from CELs. But for the Fornax PN, the total C abundance $9.02 \pm 0.07$ derived using the ORL $\lambda 4267$ line (see Table 3) is consistent within the quoted uncertainties with the value $12+\log (\mathrm{C} / \mathrm{H})=8.95$ found by Maran et al. (1984) on the basis of measurements of the ultraviolet doublet line (CEL) C III] $\lambda 1909$. A higher S/N spectrum will be needed to identify any discrepancy between the ORL and CEL abundances in PN in Fornax. 


\subsection{The central star of $P N$ in Fornax}

The Fornax PN shows a blue Wolf-Rayet bump in its spectrum: weak N III $\lambda 4640(E W=1.3 \AA, F W H M=10.4 \AA$, flux $=$ $\left.0.7 \pm 0.3 \times 10^{-16} \mathrm{erg} \mathrm{s}^{-1} \mathrm{~cm}^{-2}\right)$ and C III $\lambda 4650(E W=2.4 \AA$, $F W H M=9.7 \AA$, flux $\left.=1.2 \pm 0.3 \times 10^{-16} \mathrm{erg} \mathrm{s}^{-1} \mathrm{~cm}^{-2}\right)$, but no detectable broad He II $\lambda 4686$ and C IV $\lambda 5810$ emissions. The detected lines are insufficient for a classification as [WC] star and instead are consistent with the so-called weak emission line stars (wels: Tylenda et al. 1993). The detection of N III supports this, as this line is relatively common in wels but rare for [WC] stars. The relation between the wels and the [WC] stars it not yet understood: Gesicki et al. (2006) find the wels are most common at a stellar temperature around $50 \mathrm{kK}$, while the [WC] stars are most common at lower and higher temperatures. This suggests that the strength of the WR wind may be temperature (i.e. ionization) dependent.The derived $T_{\text {eff }}$ for the Fornax PN is consistent with the wels peak of Gesicki et al. (2006).

It is worth noting that three of the PNe in the Sagittarius dSph galaxy (Zijlstra et al. 2006) also show Wolf-Rayet features in their spectra. With the detected emissions the central star of the PN in Fornax is very similar to the central star of the PN Wray 16-423 in Sagittarius that also was classified as a wels.

The Wolf-Rayet spectral features in PNe spectra provide important constraints on the evolutionary status of the central star. As is known, central stars of PNe evolve mostly either on hydrogen-burning tracks or on helium-burning tracks, entering the white dwarf cooling phase thereafter. The hydrogenburning phase is several times longer than the helium-burning one (Vassiliadis \& Wood 1994) and therefore the probability for the central star of the PN to be detected in the helium-burning phase ranges from $20 \%$ (Iben 1984) to $25 \%$ (Schönberner 1983). But since the Wolf-Rayet central stars are known to be hydrogen-poor, with high probability such a star should be in the helium-burning phase. This could be interpreted as that for low-metallicity stars, the final AGB mass loss takes place preferentially during and immediately following a thermal pulse.

\section{Summary}

We present the highest signal-to-noise spectroscopy published to date of the only known PN in Fornax. The depth of our data permits us to measure line ratios of elements not accessible in earlier studies. We measured the electron temperature, the electron density, and element abundances for $\mathrm{He}, \mathrm{N}, \mathrm{O}, \mathrm{Ar}, \mathrm{Ne}, \mathrm{Cl}, \mathrm{C}, \mathrm{Fe}$ and $\mathrm{S}$, and derived the properties of the progenitor star. These results are presented in several tables. Except for nucleosynthetic processing within the progenitor star, these data represent a snapshot of the chemical composition in this dwarf galaxy approximately $8 \mathrm{Gyr}$ ago when the low-mass PN progenitor star was formed. Roughly at this time the overall star formation rate in Fornax reached its peak, giving rise to Fornax' predominantly intermediate-age population.

We obtain an oxygen abundance of $12+\log (\mathrm{O} / \mathrm{H})=8.28 \pm$ 0.02 . According to our analysis, this value should be corrected downward by $0.27+0.10$ dex due to the self-pollution of oxygen by the PNe progenitor. After this correction the element abundance ratios $\mathrm{Ne} / \mathrm{O}, \mathrm{S} / \mathrm{O}$ and $\mathrm{Ar} / \mathrm{O}$ appear in a good overall accord with the trends seen for $\mathrm{H}$ II regions in other galaxies. The obtained $[\mathrm{Fe} / \mathrm{H}]$ value is slightly below the spectroscopically determined mean stellar metallicity of Fornax, but this may be affected by gas-phase depletion of iron. The abundances agree well with the stellar age-metallicity relation in this galaxy. The
PN central star shows Wolf-Rayet features in its spectrum, similar to the PNe in the Sgr dSph.

Acknowledgements. We thank the anonymous referee for comments which improved the presentation of the manuscript. A.Y.K. thanks Albert Zijlstra for the enormous support, help and useful discussions. S.A.P. and A.G.P. acknowledge the partial support from Russian state program "Astronomy". E.K.G. thanks the Swiss National Science Foundation for partial support through the grants 200020-113697 and 200020-105260. This research has made use of the NASA/IPAC Extragalactic Database (NED), which is operated by the Jet Propulsion Laboratory, California Institute of Technology, under contract with the National Aeronautics and Space Administration. We have also used the Digitized Sky Survey, produced at the Space Telescope Science Institute under government grant NAG W-2166. This research has made use of NASA's Astrophysics Data System Bibliographic Services.

\section{References}

Asplund, M., Grevesse, N., Sauval, A. J., Allende Prieto, C., \& Kiselman, D. 2004, A\&A, 417, 751

Buonanno, R., Corsi, C. E., Castellani, M., et al. 1999, AJ, 118, 1671

Battaglia, G., Tolstoy, E., Helmi, A., et al. 2006, A\&A, 459, 423

Cardelli, J. A., Clayton, G. C., \& Mathis, J. S. 1989, ApJ, 345, 245

Coleman, M., Da Costa, G. S., Bland-Hawthorn, J., et al. 2004, AJ, 127, 832

Danziger, I. J., Dopita, M. A., Hawarden, T. G., \& Webster, B. L. 1978, ApJ, 220,458

Davey, A. R., Storey, P. J., \& Kisielius, R. 2000, A\&AS, 142, 85

Dinescu, D. I., Keeney, B. A., Majewski,S. R., \& Girard, T. M. 2004, AJ, 128, 687

Dudziak, G., Péquignot, D., Zijlstra, A. A., \& Walsh, J. R. 2000, A\&A, 363, 717

Esteban, C., Peimbert, M., Torres-Peimbert, S., \& Escalante, V. 1998, MNRAS, 295,401

Esteban, C., Peimbert, M., Torres-Peimbert, S., García-Rojas, J., \& Rodríguez, M. ̃. 1999, ApJS, 120, 113

Garnett, D. R. 1990, ApJ, 363, 142

Garnett, D. R. 1992, AJ, 103, 1330

Gesicki, K., Zijlstra, A. A., Acker, A., et al. 2006, A\&A, 451, 925

Grebel, E. K. 1997, Rev. Mod. Astron., 10, 29

Grebel, E. K. 1999, in The Stellar Content of the Local Group, ed. P. Whitelock, \& R. Cannon (San Francisco: ASP), IAU Symp., 192, 17

Grebel, E. K. 2000, in Star Formation from the Small to the Large Scale, ed. F. Favata, A. A. Kaas, \& A. Wilson (Noordwijk: ESA), SP-445, 87

Grebel, E. K. 2001, Ap\&SSS, 277, 231

Grebel, E. K., \& Stetson, P. B. 1999, in The Stellar Content of the Local Group, ed. P. Whitelock, \& R. Cannon (San Francisco: ASP), IAU Symp., 192, 165

Grebel, E. K., \& Gallagher, J. S. 2004, ApJ, 610, L89

Grebel, E. K., Gallagher, J. S., \& Harbeck, D. 2003, AJ, 125, 1926

Gruenwald, R., \& Viegas, S. M. 2000, ApJ, 543, 889

Guseva, N. G., Papaderos, P., Izotov, Y. I., et al. 2003, A\&A, 407, 105

Harbeck, D., Grebel, E. K., Holtzman, J., et al. 2001, AJ, 122, 3092

Iben, I. J. 1984, ApJ, 277, 333

Izotov, Y. I., \& Thuan, T. X. 1998, ApJ, 500, 188

Izotov, Y. I., \& Thuan, T. X. 1999, ApJ, 511, 639

Jacoby, G. H. 1989, ApJ, 339, 39

Kaler, J. B., \& Jacoby, G. H. 1989, ApJ, 345, 871

Kingsburg, R. L., \& Barlow, M. J. 1994, MNRAS, 271, 257

Kniazev, A. Y., Pustilnik, S. A., Masegosa, J., et al. 2000, A\&A, 357, 101

Kniazev, A. Y., Grebel, E. K., Hao, L., et al. 2003, ApJ, 593, L73

Kniazev A. Y., Pustilnik S. A., Grebel, E. K., Pramskij A. G., \& Lee, H. 2004, ApJS, 153, 429

Kniazev A. Y., Grebel, E. K., Pustilnik S. A., Pramskij A. G., \& Zucker, D. 2005a, AJ, 130, 1558

Kniazev, A. Y., Grebel, E. K., Zucker, D., et al. 2005b, in Planetary Nebulae as Astronomical Tools, AIP Conf. Proc., ed. G. Stasińska, \& R. Szczerba, 804, 15

Koch, A., Grebel, E. K., Wyse, R. F. G., et al. 2006, AJ, 131, 895

Koch, A., Grebel, E. K., Kleyna, J. T., et al. 2007, AJ, 133, 270

Liu, X.-W. 2003, in Planetary Nebulae, ed. S. Kwok, M. Dopita, \& R. Sutherland (San Francisco: ASP), IAU Symp., 209, 339

Liu, X.-W., Storey, P. J., Barlow, M. J., et al. 2000, MNRAS, 312, 585

Maran, S. P., Gull, T. R., Stecher, T. P., Aller, L. H., \& Keyes C. D. 1984, ApJ, 280,615

Marigo, P. 2001, A\&A, 370, 194

Mateo, M., Olszewski, E., Welch, D., Fischer, P., \& Kunkel, W. 1991, AJ, 102, 914

Mateo, M. 1998, ARA\&A, 36, 435 
Lanfranchi, G. A., \& Matteucci, F. 2004, MNRAS, 351, 1338

Péquignot, D., Walsh, J. R., Zijlstra, A. A., \& Dudziak, G. 2000, A\&A, 361, L1

Pont, F., Zinn, R., Gallart, C., Hardy, E., \& Winnick, R. 2004, AJ, 127, 840

Rauch, T. 2003, A\&A, 403, 709

Richer, M. G., \& McCall, M. L. 1995, ApJ, 445, 642

Richer, M. G., \& Lopez, J. A. 2005, in Planetary nebulae as tools of astrophysics, AIP Conf. Proc., 804, 293

Richer, M. G., McCall, M. L., \& Stasińska, G. 1998, A\&A, 340, 67

Rodríguez, M., \& Rubin, R. H. 2005, ApJ, 626, 900

Saviane, I., Held, E. V., \& Bertelli, G. 2000, A\&A, 355, 56

Schlegel, D., Finkbeiner, D., \& Davis, M. 1998, ApJ, 500, 525

Schönberner, D. 1983, ApJ, 272, 708

Shapley, H. 1938, Nature, 142, 715

Shetrone, M. D., Côté, P., \& Sargent, W. L. W. 2001, ApJ, 548, 592
Stasińska, G. 1990, A\&AS, 83, 501

Stetson, P. B., Hesser, J. E., \& Smecker-Hane, T. A. 1998, PASP, 110, 533

Stoughton, C., et al. 2001, AJ, 123, 485

Tolstoy, E., Venn, K., Shetrone, M., et al. 2003, AJ, 125, 707

Torres-Peimbert, S., \& Peimbert, M. 1977, RMxAA, 2, 181

Tylenda, R., Acker, A., \& Stenholm, B. 1993, A\&AS, 102, 595

Vassiliadis, E., \& Wood, P. R. 1993, ApJ, 413, 641

Vassiliadis, E., \& Wood, P. R. 1994, ApJS, 92, 125

Walker, M. G., Mateo, M., Olszewski, E. W., et al. 2006, AJ, 131, 2114

Walsh, J. R., Dudziak, G., Minniti, D., \& Zijlstra, A. A. 1997, ApJ, 487, 651

York, D. G., Adelman, J., Anderson, J. E., et al. 2000, AJ, 120, 1579

Zijlstra, A. A., \& Walsh, J. R. 1986, A\&A, 312, L21

Zijlstra, A. A., \& Pottasch, S. R. 1989, A\&A, 216, 245

Zijlstra, A. A., Gesicki, K., Walsh, J. R., et al. 2006, MNRAS, 369, 875 